\title{
Diagnostics for Linear Models With Functional Responses
}

\author{
Qing Shen \\ Edmunds.com Inc. \\ 2401 Colorado Ave., Suite 250 \\ Santa Monica, CA 90404 \\ (shenqing26@hotmail.com)
}

\author{
Hongquan Xu \\ Department of Statistics \\ University of California \\ Los Angeles, CA 90095-1554 \\ (hqxu@stat.ucla.edu)
}

(June 19, 2006)

\begin{abstract}
Linear models where the response is a function and the predictors are vectors are useful in analyzing data from designed experiments and other situations with functional observations. Residual analysis and diagnostics are considered for such models. Studentized residuals are defined and their properties are studied. Chi-square quantile-quantile plots are proposed to check the assumption of Gaussian error process and outliers. Jackknife residuals and an associated test are proposed to detect outliers. Cook's distance is defined to detect influential cases. The methodology is illustrated by an example from a robust design study.
\end{abstract}

KEY WORDS: Cook's distance; Functional F test; Functional regression analysis; Jackknife residual; Studentized residual.

\section{INTRODUCTION}

Functional data analysis becomes increasingly popular as modern technology enables relatively easy access to data collected continuously over a period of time. Because only a finite number of observations are recorded, one may apply traditional multivariate analysis or longitudinal data analysis strategies. However, it is often more natural and convenient to think of, model and analyze the observed functional data as single elements, rather than merely a sequence of individual (repeated) observations as pointed out by Ramsay and Dalzell (1991). Ramsay and Silverman (2005) provided introductions to various issues on functional data analysis; Ramsay and Silverman (2002) provided applications in criminology, meteorology, medicine, and many other fields.

Linear models are useful in describing and predicting responses by a set of predictors. In a functional linear model, either the response or the predictors or both could be functions. In this paper we assume that $\mathbf{Y}(t)=\mathbf{X} \boldsymbol{\beta}(t)+\boldsymbol{\epsilon}(t)$ where the responses are functions and the predictors are scalar vectors. Such linear models, including functional analysis of variance as a special case, have been studied by many authors; see, for example, Ramsay and Silverman (2005), Faraway (1997), Fan 
and Lin (1998), Eubank (2000), and Shen and Faraway (2004). Ramsay and Silverman (2005) laid out some general ideas on estimation and provided preliminary methods for inference. Faraway (1997) pointed out the inappropriateness of traditional multivariate test statistics, proposed a bootstrap-based testing method and discussed residual analysis for such models. Fan and Lin (1998) proposed adaptive transform-based tests for functional analysis of variance models. Eubank (2000) considered tests for a constant mean function using a cosine basis function approach. Shen and Faraway (2004) recently proposed a functional F test for comparing nested functional linear models. We shall point out that many data from designed experiments fit well with such functional linear models. For example, Nair, Taam, and Ye (2002) used functional linear models to analyze some data from robust design studies.

Outliers and influential cases can be frequently found not only in observational studies but also in designed experiments. Including them in the analysis often leads to misleading conclusions. However, diagnostics have been largely ignored for functional linear models in the literature, most possibly due to lack of proper tools. Since no formal procedures are available, the current practice in functional regression analysis is to skip diagnostics completely or to detect outliers visually; for example, Faraway (1997) and Shen and Faraway (2004) removed an obvious outlier from their data. The purpose of this paper is to formally define diagnostic statistics for functional linear regression models with fixed covariates and to provide simple computational methods, which makes it possible to automate the diagnostic checking procedure.

In Section 2, we briefly review functional linear models and a functional $\mathrm{F}$ test proposed by Shen and Faraway (2004). In Section 3, we define studentized residuals for residual analysis and propose chi-square quantile-quantile (Q-Q) plots to check the assumption of Gaussian error process and outliers. We then define jackknife residuals and develop a formal test to detect outliers. We also define Cook's distance to detect influential cases. Formulas for easy computation of jackknife residuals and Cook's distance are given. The methodology is illustrated by an example from a robust design study in Section 4. Concluding remarks are given in Section 5.

\section{FUNCTIONAL LINEAR MODELS}

Suppose we have functional response data $y_{i}(t), i=1, \ldots n, t \in[a, b]$. We are interested in building a regression model to relate this response to a vector of predictors $\mathbf{x}_{i}=\left(x_{i 1}, \ldots, x_{i p}\right)^{T}$. The model 
takes the familiar form

$$
y_{i}(t)=\mathbf{x}_{i}^{T} \boldsymbol{\beta}(t)+\epsilon_{i}(t)
$$

where $\boldsymbol{\beta}(t)=\left(\beta_{1}(t), \ldots, \beta_{p}(t)\right)^{T}$ are unknown parameter functions, and $\epsilon_{i}(t)$ is a Gaussian stochastic process with mean zero and a covariance function $\gamma(s, t)$. We assume $\epsilon_{i}(\cdot)$ and $\epsilon_{j}(\cdot)$ are independent for $i \neq j$.

The unknown functions $\boldsymbol{\beta}(t)$ can be estimated by minimizing $\sum_{i=1}^{n}\left\|y_{i}-\mathbf{x}_{i}^{T} \boldsymbol{\beta}\right\|^{2}$, where $\|f\|=$ $\left(\int f(t)^{2} d t\right)^{1 / 2}$ is the $L_{2}$ norm of function $f=f(t)$. This minimization leads to the least squares estimates $\hat{\boldsymbol{\beta}}(t)=\left(\mathbf{X}^{T} \mathbf{X}\right)^{-1} \mathbf{X}^{T} \mathbf{Y}(t)$, where $\mathbf{X}=\left(\mathbf{x}_{1}, \ldots, \mathbf{x}_{n}\right)^{T}$ is the usual $n \times p$ model matrix while $\mathbf{Y}(t)=\left(y_{1}(t), \ldots, y_{n}(t)\right)^{T}$ is a vector of responses. The predicted (or fitted) responses are $\hat{y}_{i}(t)=\mathbf{x}_{i}^{T} \hat{\boldsymbol{\beta}}(t)$ and the residuals are $\hat{\epsilon}_{i}(t)=y_{i}(t)-\hat{y}_{i}(t)$. The residual sum of squares is $r s s=$ $\sum_{i=1}^{n}\left\|\hat{\epsilon}_{i}\right\|^{2}=\sum_{i=1}^{n} \int \hat{\epsilon}_{i}(t)^{2} d t$.

An important inference problem is to compare two nested linear models, $\omega$ and $\Omega$, where $\omega$ has $q$ parameter functions, $\Omega$ has $p$ parameter functions, and $\omega$ results from a linear restriction on the parameter functions of $\Omega$. A naive approach is to examine the point-wise $t$ statistics on each time point for testing $\boldsymbol{\beta}(t)$. This carries a serious problem with multiple-comparison and if Bonferroni corrections were applied to the significance level, power would be significantly compromised because the responses are often highly correlated within each unit. Faraway (1997) and Ramsey and Silverman (2005) proposed bootstrap-based and permutation-based tests, which require intensive computation. As pointed out by Faraway (1997), traditional multivariate test statistics are inappropriate due to the influence of unimportant variation directions.

To overcome these issues, Shen and Faraway (2004) proposed a functional F test. Define

$$
F=\frac{\left(r s s_{\omega}-r s s_{\Omega}\right) /(p-q)}{r s s_{\Omega} /(n-p)}
$$

where $r s s_{\omega}$ and $r s s \Omega$ are residual sum of squares under models $\omega$ and $\Omega$, respectively. When the null model is true, this functional $\mathrm{F}$ statistic is distributed like a ratio of two linear combinations of infinite independent $\chi^{2}$ random variables, that is, $\left[(p-q)^{-1} \sum_{k=1}^{\infty} \lambda_{k} \chi_{k}^{2}(p-q)\right] /[(n-$ $\left.p)^{-1} \sum_{k=1}^{\infty} \lambda_{k} \chi_{k}^{2}(n-p)\right]$, where $\lambda_{1} \geq \lambda_{2} \geq \ldots \geq 0$ are eigenvalues of the covariance function $\gamma(s, t)$, $\chi_{k}^{2}(a)$ is a $\chi^{2}$ random variable with $a$ degrees of freedom, and all the $\chi^{2}$ random variables are independent of each other. The exact distribution is too complicated for practical use. Shen and Faraway (2004) suggested to use the Satterthwaite (1941, 1946) approximation and showed that it can be effectively approximated by an ordinary $\mathrm{F}$ distribution with degrees of freedoms 
$d f_{1}=\lambda(p-q)$ and $d f_{2}=\lambda(n-p)$, where

$$
\lambda=\left(\sum_{k=1}^{\infty} \lambda_{k}\right)^{2} / \sum_{k=1}^{\infty} \lambda_{k}^{2}
$$

is called the degrees-of-freedom-adjustment-factor by Shen and Faraway (2004).

Model selection is an important issue in regression analysis. Stepwise model selection requires an easy way of calibrating the p-value of a predictor in the full model, i.e., to test $\beta_{j}(t) \equiv 0$. This can be done by fitting a reduced model without the $j$ th covariate and using the functional $\mathrm{F}$ test statistic

$$
F_{j}=\frac{r s s_{j}-r s s}{r s s /(n-p)}
$$

where $r s s_{j}$ is the residual sum of squares under $\beta_{j}(t) \equiv 0$. Shen and Faraway (2004) showed that it is indeed unnecessary to fit the reduced model, because $F_{j}$ can be derived from quantities obtained directly from the fitting of the full model, i.e.,

$$
F_{j}=\frac{\left\|\hat{\beta}_{j}\right\|^{2}}{(r s s /(n-p))\left(\mathbf{X}^{T} \mathbf{X}\right)_{j j}^{-1}}=\frac{\int \hat{\beta}_{j}^{2}(t) d t}{(r s s /(n-p))\left(\mathbf{X}^{T} \mathbf{X}\right)_{j j}^{-1}},
$$

where $\left(\mathbf{X}^{T} \mathbf{X}\right)_{j j}^{-1}$ is the $j$ th diagonal element of $\left(\mathbf{X}^{T} \mathbf{X}\right)^{-1}, \hat{\beta}_{j}(t)$ is the estimate of $\beta_{j}(t)$, and rss is the residual sum of squares under the full model. The null distribution of the functional $\mathrm{F}$ statistic $F_{j}$ can be approximated by an ordinary $\mathrm{F}$ distribution with degrees of freedom $d f_{1}=\lambda$ and $d f_{2}=\lambda(n-p)$, where $\lambda$ is the degrees-of-freedom-adjustment-factor defined in (3).

In practice, we do not observe $y_{i}(t)$ for all $t$, but only $y_{i}\left(t_{i j}\right)$. It is desirable to collect data at fixed time points $t_{1}, \ldots, t_{m}$ for easy interpretation and estimation. This happens in many designed experiments or studies. It is possible that the $t_{i j}$ are different for different $i$. In such a case, smoothing techniques can be used to get fixed time points; see Faraway (1997). Here we simply assume that the responses are observed on evenly spaced fixed time points $t_{1}, \ldots, t_{m}$. Then, (1) becomes

$$
y_{i}\left(t_{j}\right)=\mathbf{x}_{i}^{T} \boldsymbol{\beta}\left(t_{j}\right)+\epsilon\left(t_{j}\right), \text { for } i=1, \ldots, n, j=1, \ldots, m,
$$

and we can do point-wise estimation and regression. We shall replace the integration with summation, compute $\left\|\hat{\epsilon}_{i}\right\|^{2}=\sum_{k=1}^{m} \hat{\epsilon}_{i}\left(t_{k}\right)^{2} / m$ and $\left\|\hat{\beta}_{j}\right\|^{2}=\sum_{k=1}^{m} \hat{\beta}_{j}\left(t_{k}\right)^{2} / m$. We can estimate the covariance function $\gamma(s, t)$ by the empirical covariance matrix $\hat{\boldsymbol{\Sigma}}=\left(\sum_{i=1}^{n} \hat{\epsilon}_{i}\left(t_{j}\right) \hat{\epsilon}_{i}\left(t_{k}\right) /(n-p)\right)_{m \times m}$ and estimate the degrees-of-freedom-adjustment-factor by

$$
\hat{\lambda}=\operatorname{trace}(\hat{\boldsymbol{\Sigma}})^{2} / \operatorname{trace}\left(\hat{\boldsymbol{\Sigma}}^{2}\right) .
$$

Large degrees of freedom (say $n-p \geq 30$ ) are desired for a good estimation of $\lambda$. 


\section{DIAGNOSTICS}

Diagnostics are as important for functional regression as for scalar regression. We shall use residuals to check various assumptions and to identify potential outliers and influential cases.

A straightforward approach is to perform point-wise diagnostics. Point-wise residual plots and normal Q-Q plots are useful to detect certain violations of assumptions and outliers. However, this approach ignores the fact that the residuals are correlated over time $t$. In the spirit of the functional $\mathrm{F}$ test, we shall develop diagnostic procedures for functional regression that view each curve as a point in a functional space.

\subsection{Studentized Residuals}

Let $\mathbf{H}=\mathbf{X}\left(\mathbf{X}^{T} \mathbf{X}\right)^{-1} \mathbf{X}^{T}$ be the hat matrix. The leverage $h_{i i}$ for the $i$ th case is the $i$ th diagonal element of $\mathbf{H}$. For fixed $t$, the standard results in scalar regression show that $\operatorname{var}\left(\hat{\epsilon}_{i}(t)\right)=(1-$ $\left.h_{i i}\right) \gamma(t, t)$. Therefore,

$$
E\left(\left\|\hat{\epsilon}_{i}\right\|^{2}\right)=E \int \hat{\epsilon}_{i}(t)^{2} d t=\int E\left(\hat{\epsilon}_{i}(t)^{2}\right) d t=\left(1-h_{i i}\right) \int \gamma(t, t) d t=\left(1-h_{i i}\right) \Delta^{2} .
$$

where $\Delta^{2}=\int \gamma(t, t) d t$ is the total variance of the error process. Because $\sum_{i=1}^{n}\left(1-h_{i i}\right)=n-p$, $\hat{\Delta}^{2}=r s s /(n-p)$ is an unbiased estimate of $\Delta^{2}$. So we formally define (internally) $L_{2}$ studentized residuals as

$$
S_{i}=\frac{\left\|\hat{\epsilon}_{i}\right\|}{\sqrt{1-h_{i i}} \hat{\Delta}}=\frac{\sqrt{\int \hat{\epsilon}_{i}^{2}(t) d t}}{\sqrt{1-h_{i i}} \sqrt{r s s /(n-p)}} .
$$

The $L_{2}$ studentized residuals can be used in a similar way as in scalar regression. For example, we can make a residuals versus fitted plot by plotting $S_{i}$ against $\left\|\hat{y}_{i}\right\|$. Such a plot can be used to check the assumption of equal total variance and to detect potential outliers. As in scalar regression, if the residuals follow the same error process, $S_{i}$ and $\left\|\hat{y}_{i}\right\|$ are uncorrelated; therefore, we expect to see no relationship between $S_{i}$ and $\left\|\hat{y}_{i}\right\|$.

To check the assumption of Gaussian process, we shall study the distribution of the residuals. We have this important result, which can be proved similarly as Theorem 1 of Shen and Faraway (2004).

Theorem 1. If the error process is Gaussian, $\left\|\hat{\epsilon}_{i}\right\|^{2}$ is distributed like a linear combination of infinite independent chi-square random variables with 1 degree of freedom, that is,

$$
\left\|\hat{\epsilon}_{i}\right\|^{2} \sim\left(1-h_{i i}\right) \sum_{k=1}^{\infty} \lambda_{k} \chi_{k}^{2}(1),
$$


where $\lambda_{k}$ are eigenvalues of the covariance function $\gamma(s, t)$ and all the $\chi^{2}$ random variables are independent of each other.

The above result indicates that $\left\|\hat{\epsilon}_{i}\right\|^{2} /\left(1-h_{i i}\right)$ are identically distributed. Using the Satterthwaite approximation, $\left\|\hat{\epsilon}_{i}\right\|^{2} /\left(1-h_{i i}\right)$ are approximately distributed as $c \chi^{2}(\lambda)$, where $c$ is a constant and $\lambda$ is the adjustment factor defined in (3). Therefore, to check whether the errors are Gaussian, we shall estimate $\lambda$ by (5) and make a chi-square Q-Q plot by plotting $S_{i}^{2}$ against quantiles of a $\chi^{2}$ distribution with $\hat{\lambda}$ degrees of freedom. If the model is correct and the Gaussian assumption holds, the points should be close to a straight line.

\subsection{Outliers and Jackknife Residuals}

As in the context of scalar regression, we define jackknife residuals for functional regression to detect outliers.

Suppose that the $i$ th case is a suspected outlier. We shall delete the $i$ th case from the data and use the remaining $n-1$ cases to fit the linear model. Let $\hat{\boldsymbol{\beta}}_{(i)}(t)$ be the estimate of $\boldsymbol{\beta}(t)$ and $r s s_{(i)}$ be the residual sum of squares, computed without the $i$ th case. Let $\mathbf{X}_{(i)}$ and $\mathbf{Y}_{(i)}(t)$ be the $\mathbf{X}$ matrix and the $\mathbf{Y}(t)$ vector with the $i$ th row deleted. Then, $\hat{\boldsymbol{\beta}}_{(i)}(t)=\left(\mathbf{X}_{(i)}^{T} \mathbf{X}_{(i)}\right)^{-1} \mathbf{X}_{(i)}^{T} \mathbf{Y}_{(i)}(t)$.

For the deleted case, compute the fitted curve $\tilde{y}_{i}(t)=\mathbf{x}_{i}^{T} \hat{\boldsymbol{\beta}}_{(i)}(t)$. Since the $i$ th case is not used in estimation, $y_{i}(t)$ and $\tilde{y}_{i}(t)$ are independent for fixed $t$. The variance of $y_{i}(t)-\tilde{y}_{i}(t)$ is

$$
\operatorname{var}\left(y_{i}(t)-\tilde{y}_{i}(t)\right)=\gamma(t, t)+\gamma(t, t) \mathbf{x}_{i}^{T}\left(\mathbf{X}_{(i)}^{T} \mathbf{X}_{(i)}\right)^{-1} \mathbf{x}_{i}
$$

If the $i$ th case is not an outlier, $E\left(y_{i}(t)-\tilde{y}_{i}(t)\right) \equiv 0$. Then,

$$
E\left\|y_{i}-\tilde{y}_{i}\right\|^{2}=E \int\left(y_{i}(t)-\tilde{y}_{i}(t)\right)^{2} d t=\int E\left(y_{i}(t)-\tilde{y}_{i}(t)\right)^{2} d t=\left(1+\mathbf{x}_{i}^{T}\left(\mathbf{X}_{(i)}^{T} \mathbf{X}_{(i)}\right)^{-1} \mathbf{x}_{i}\right) \int \gamma(t, t) d t .
$$

We estimate $\Delta^{2}=\int \gamma(t, t) d t$ by $\hat{\Delta}_{(i)}^{2}=r s s_{(i)} /(n-p-1)$. So we define $L_{2}$ jackknife (or externally studentized) residuals as

$$
J_{i}=\frac{\left\|y_{i}-\tilde{y}_{i}\right\|}{\sqrt{1+\mathbf{x}_{i}^{T}\left(\mathbf{X}_{(i)}^{T} \mathbf{X}_{(i)}\right)^{-1} \mathbf{x}_{i}} \hat{\Delta}_{(i)}}=\frac{\sqrt{\int\left(y_{i}(t)-\tilde{y}_{i}(t)\right)^{2} d t}}{\sqrt{1+\mathbf{x}_{i}^{T}\left(\mathbf{X}_{(i)}^{T} \mathbf{X}_{(i)}\right)^{-1} \mathbf{x}_{i}} \sqrt{r s s_{(i)} /(n-p-1)}} .
$$

To derive the distribution of $J_{i}$, we shall take an alternative approach and consider the so called mean shift outlier model. Suppose that the $i$ th case is a candidate for an outlier. Assume that the model for all other cases is

$$
y_{j}(t)=\mathbf{x}_{j}^{T} \boldsymbol{\beta}(t)+\epsilon_{j}(t), \quad j \neq i,
$$


but for case $i$, the model is

$$
y_{i}(t)=\mathbf{x}_{i}^{T} \boldsymbol{\beta}(t)+\delta(t)+\epsilon_{i}(t) .
$$

Then testing whether the $i$ th case is an outlier is equivalent to testing $\delta(t) \equiv 0$. We can create a new predictor variable, say $u$, with $u_{i}=1$ and $u_{j}=0$ for $j \neq i$. By (4), the functional $\mathrm{F}$ test for $\delta(t) \equiv 0$ is

$$
F_{i}=\frac{\|\hat{\delta}\|^{2}}{(n-p-1)^{-1} r \tilde{s} s\left(\tilde{\mathbf{X}}{ }^{T} \tilde{\mathbf{X}}\right)_{i i}^{-1}},
$$

where $\hat{\delta}(t)$ is the estimate of $\delta(t), r \tilde{s} s$ is the residual sum of squares, and $\tilde{\mathbf{X}}$ is the model matrix under the mean shift outlier model. It follows from scalar regression that $\hat{\delta}(t)=y_{i}(t)-\tilde{y}_{i}(t)$, $r \tilde{s} s=r s s_{(i)}$ and $\left(\tilde{\mathbf{X}}^{T} \tilde{\mathbf{X}}\right)_{i i}^{-1}=\left(1-h_{i i}\right)^{-1}$; see, for example, Sen and Srivastava (1990, p. 174-175). It is known in scalar regression (see Weisberg 1985 p. 293) that

$$
1+\mathbf{x}_{i}^{T}\left(\mathbf{X}_{(i)}^{T} \mathbf{X}_{(i)}\right)^{-1} \mathbf{x}_{i}=\left(1-h_{i i}\right)^{-1}
$$

Comparing (7) and (8) yields $F_{i}=J_{i}^{2}$. When $\delta(t) \equiv 0$, the statistic $F_{i}$ defined in (8) has a functional F distribution according to Shen and Faraway (2004). The next theorem summarizes the results.

Theorem 2. If the ith case is not an outlier, $J_{i}^{2}$ is distributed like a ratio of two linear combinations of infinite independent chi-square random variables, that is,

$$
J_{i}^{2} \sim \frac{\sum_{k=1}^{\infty} \lambda_{k} \chi_{k}^{2}(1)}{(n-p-1)^{-1} \sum_{k=1}^{\infty} \lambda_{k} \chi_{k}^{2}(n-p-1)}
$$

where $\lambda_{k}$ are eigenvalues of the covariance function $\gamma(s, t)$ and all the $\chi^{2}$ random variables are independent of each other.

In practice, we use the Satterthwaite approximation and approximate this functional F distribution by an ordinary $\mathrm{F}$ distribution with degrees of freedom $d f_{1}=\lambda$ and $d f_{2}=\lambda(n-p-1)$, where $\lambda$ is the adjustment factor defined in (3). We can estimate $\lambda$ by (5) and do an F test to formally detect outliers. Since the test is usually done after looking at the results and applied for all cases, adjustment of the significance level such as Bonferroni method should be applied.

Furthermore, to avoid fitting the regression model with a case deleted for $n$ times, as in scalar regression, jackknife residuals can be computed directly from studentized residuals and leverages as follows:

$$
J_{i}=S_{i} \sqrt{\frac{n-p-1}{n-p-S_{i}^{2}}} .
$$

The proof is given in the Appendix. 


\subsection{Influential Cases and Cook's Distance}

Cook's distance is useful for identifying influential cases in scalar regression. Here we extend the definition to functional regression.

To determine whether the $i$ th case is influential, we can measure the influence by comparing $\hat{\boldsymbol{\beta}}(t)$ to $\hat{\boldsymbol{\beta}}_{(i)}(t)$, the estimates of $\boldsymbol{\beta}(t)$ with and without the $i$ th case. Formally, define $L_{2}$ Cook's distance as

$$
D_{i}=\frac{\int\left(\hat{\boldsymbol{\beta}}_{(i)}(t)-\hat{\boldsymbol{\beta}}(t)\right)^{T}\left(\mathbf{X}^{T} \mathbf{X}\right)\left(\hat{\boldsymbol{\beta}}_{(i)}(t)-\hat{\boldsymbol{\beta}}(t)\right) d t}{p \cdot r s s /(n-p)} .
$$

Alternatively, if we define $\hat{\mathbf{Y}}(t)=\mathbf{X} \hat{\boldsymbol{\beta}}(t)$ and $\hat{\mathbf{Y}}_{(i)}(t)=\mathbf{X} \hat{\boldsymbol{\beta}}_{(i)}(t)$, then (11) can be rewritten as

$$
D_{i}=\frac{\int\left(\hat{\mathbf{Y}}_{(i)}(t)-\hat{\mathbf{Y}}(t)\right)^{T}\left(\hat{\mathbf{Y}}_{(i)}(t)-\hat{\mathbf{Y}}(t)\right) d t}{p \cdot r s s /(n-p)}
$$

which measures the distance between $\hat{\mathbf{Y}}(t)$ and $\hat{\mathbf{Y}}_{(i)}(t)$, the fitted responses with and without the $i$ th case. Cases for which $D_{i}$ are large have substantial influence on $\hat{\boldsymbol{\beta}}(t)$ and on fitted responses, and the deletion of them may result in important changes in conclusions. As in scalar regression, it can be shown (see Appendix) that Cook's distance can be computed directly from the studentized residual and leverage as follows:

$$
D_{i}=\frac{1}{p} \frac{h_{i i}}{1-h_{i i}} S_{i}^{2}
$$

This formula shall save us great amount of computation time since there is no need to fit $n$ models, each with a case deleted. By (13), a highly influential case must have a large leverage or a large studentized residual. An influential case may, but not necessarily, be an outlier.

\section{AN EXAMPLE}

\subsection{A Robust Design Experiment}

For illustration, we use one of the experiments reported by Nair et al. (2002). A robust parameter design experiment was conducted to study the effects of 7 process assembly parameters (factors $A-G)$ on the audible noise levels of alternators. The experiment used a $2_{I V}^{7-2}$ design with defining relation $I=C E F G=A B C D F=A B D E G$. For each experimental combination, 43 measurements of sound pressure levels (responses) were recorded at rotating speeds ranging from 1000 to 2500 revolutions per minute, where the rotating speed was a signal factor. Figure 1 shows the 32 observed (and fitted) response curves. The original data include four additional replications collected at the 
Table 1: F Statistics and P Values for the Main Effects Model

\begin{tabular}{cccccccc}
\hline \hline & $A$ & $B$ & $C$ & $D$ & $E$ & $F$ & $G$ \\
\hline F Value & 3.27 & 1.15 & 3.61 & 5.46 & 0.91 & 1.01 & 7.81 \\
P-Value & .0093 & .3388 & .0051 & .0002 & .4762 & .4117 & .0000 \\
\hline
\end{tabular}

Table 2: F Statistics and P Values for the Reduced Model

\begin{tabular}{ccccc}
\hline \hline & $A$ & $C$ & $D$ & $G$ \\
\hline F Value & 3.26 & 3.60 & 5.45 & 7.79 \\
P-Value & .0076 & .0040 & .0001 & .0000 \\
\hline
\end{tabular}

high levels of all factors, which we do not use here. For further description of the data, see Nair et al. (2002).

For ease of illustration, we first fit a main effects model (indeed none of the two-factor interactions is significant). The low and high levels are coded as -1 and +1 , respectively. Table 1 gives the F statistics and p-values. The residual sum of squares is 273.09 and estimated adjustment factor is 4.81. Note that $\hat{\lambda}(n-p)=(4.81)(32-8)=115.44$. The p-value of effect $A$ is computed as the upper tail probability of 3.27 under an ordinary $\mathrm{F}$ distribution with degrees of freedom 4.81 and 115.44. Other p-values are obtained similarly. The p-values show that $D$ and $G$ are very significant, $C$ and $A$ are significant at the $1 \%$ margin.

We then simplify the main effects model by dropping insignificant terms. Table 2 shows the F statistics and p-values for a reduced model with main effects $A, C, D$ and $G$. As expected, all effects are now significant at the 1\% level. The residual sum of squares is 308.01 and the estimated adjustment factor is 5.14. The p-values in Table 2 are computed using this new adjustment factor.

We compare the reduced model with the main effects model by performing a functional $\mathrm{F}$ test as follows. The F statistic in $(2)$ is 1.02 , with $d f_{1}=(4.81)(8-5)=14.43$ and $d f_{2}=(4.81)(32-8)=$ 115.44, yielding a p-value of 0.44 . So we accept the reduced model with 4 main effects and proceed to diagnostics.

Figure 2 shows the diagnostics plots for the reduced model. Both the residuals versus fitted plot and chi-square Q-Q plot suggest a potential outlier. The jackknife residuals plot and Cook's distance plot confirm that case 16 have the largest jackknife residual and Cook's distance. The formal $\mathrm{F}$ test with Bonferoni adjustment declares that case 16 is an outlier at the $5 \%$ level. The 
Table 3: F Statistics and P Values for the Reduced Model Without Case 16

\begin{tabular}{ccccc}
\hline \hline & $A$ & $C$ & $D$ & $G$ \\
\hline F Value & 2.47 & 5.29 & 4.29 & 10.48 \\
P-Value & .0256 & .0001 & .0005 & .0000 \\
\hline
\end{tabular}

jackknife residual for case 16 is just above the critical value (the horizontal line on the jackknife plot) at the $5 \%$ level. Notice that there is a big gap between the observed and fitted response curves for case 16 in Figure 1.

We repeat the above analysis without case 16 and still conclude that $A, C, D$ and $G$ are significant. Table 3 gives the $\mathrm{F}$ statistics and p-values. The residual sum of squares is 265.95 and estimated adjustment factor is 6.06. Note that $G$ is still most significant, but $C$ is more significant than $D$, and $A$ becomes less significant (p-value increases from .0076 to .0256). This time diagnostics plots do not show apparent patterns calling for attention.

\subsection{Simulation Study of Size and Power}

Shen and Faraway (2004) used simulation to study the size and power of the functional F test in comparison with multivariate likelihood ratio test and B-spline based test under similar conditions to their ergonomics data. Their simulation suggests that the functional $\mathrm{F}$ test has a fairly accurate size and could be quite powerful for some type of covariance structure. Notice that our data are much rougher than their ergonomics data. It is of interest to investigate how the $\mathrm{F}$ test and diagnostics perform for our data.

We simulated response curves as the weighted average of the predicted curves from the reduced model and the main effects model plus Gaussian errors with mean zero and covariance $\hat{\boldsymbol{\Sigma}}$, the empirical covariance matrix from the reduced model without case 16 . The weight ran from 0 (corresponding to the reduced model) to 1 (corresponding to the main effects model) in increments of 0.1. Note that traditional multivariate tests cannot be applied here because there are 43 dimensions and only 32 cases. For comparison, we also applied B-spline based tests, where the likelihood ratio test was applied on the B-spline expansion coefficients. Figure 3 shows the powers (i.e., probabilities of rejecting the reduced model) of the $\mathrm{F}$ test and likelihood ratio tests with 5 and 8 B-spline basis functions at significance level 0.05 over 10,000 repetitions. The $\mathrm{F}$ test was comparable to the likelihood ratio test with 5 B-spline basis functions: F test had a slightly lower power when weight 
was less than 0.6 and a higher power when weight was more than 0.6. The likelihood ratio test with 8 B-spline basis functions was the most powerful among the three tests. We actually computed all likelihood ratio tests with 4 to 10 B-spline basis functions, among which the test with 8 B-spline basis functions was the most powerful one.

The size of the F test (i.e., the power at weight $=0$ ) was 0.041 , which was below the pre-specified significance level 0.05, indicating that the $\mathrm{F}$ test was conservative here. The reason was that the degrees of freedom adjustment factor was underestimated in the simulation due to a relative small sample size. The estimated adjustment factor had an average of 4.90 and standard deviation of 0.59 whereas the true adjustment factor used in simulation was 6.06. This was not bad at all, considering that only $32-8=24$ degrees of freedom were available to estimate a $43 \times 43$ covariance matrix. Since we knew the true eigenvalues, we also applied the F test using the true adjustment factor 6.06. With this modification, the $\mathrm{F}$ test had a size of 0.053 and almost the same power as the likelihood ratio test with 8 B-spline basis functions.

We conducted another simulation to investigate the performance of diagnostic procedures. For case 16, we simulated response curve as the weighted average of the observed and the predicted from the reduced model (without case 16) while for all other cases, response curves were simply the predicted curves from the reduced model (without case 16), and added Gaussian errors from the empirical covariance matrix as before. Then we computed jackknife residuals and applied the F test to see whether case 16 was identified as an outlier at significance level 0.05 over 10,000 simulated data sets. The power curve was similar to that of the $\mathrm{F}$ test in Figure 3, which was consistent with the theory developed in Section 3. The simulated size was 0.041 and the power at weight=1 was 0.949. As in the previous simulation, the size was underestimated due to the fact that adjustment factor was underestimated (the average was 5.01 and standard deviation was 0.71). An F test using the true adjustment factor had a size of 0.051 and a power of 0.962 when weight $=1$.

\subsection{Discussion}

Faraway (1997) suggested three kinds of plots for residual analysis. The first kind is plots of the estimated eigenfunctions and their associated eigenvalues. These plots show the nature of the unexplained variation in the model and are potentially useful for understanding the error process. Figure 4 shows the first 8 estimated eigenfunctions and their associated eigenvalues from the reduced model without case 16 . The 8 eigenfunctions explain $90 \%$ variation of the residual functions. The plots indicate that the error process is rather complicated. It would be difficult to decide the 
dimension of the error process. The functional $\mathrm{F}$ test does not suffer from this difficulty, which is an advantage over tests based on basis expansion coefficients.

The second kind is normal Q-Q plots of the estimated scores of each residual curve. These plots are useful for detecting outliers and for assessing the assumption of Gaussian error process. Typically we only need examine a few of these plots that associate with the leading eigenvalues. These plots should be examined if the chi-square Q-Q plot indicates any problems.

The third kind is plots of residuals versus fitted for each time point $t_{j}$. As in scalar regression, these plots are useful for checking model assumptions and outliers. But it is sometimes difficult to detect outliers when the patterns are not consistent across all time points. For our example, there are 43 plots to be examined and many (but not all) plots show that case 16 is a potential outlier. Our diagnostics plots show clearly that case 16 is a potential outlier.

\section{CONCLUDING REMARKS}

Treating each response curve as a point in an $L_{2}$ functional space, residual analysis and diagnostics are studied for linear models where the response is a function and the predictors are vectors. Studentized residuals, jackknife residuals and Cook's distance in the $L_{2}$ sense are defined similarly as their counterparts in scalar regression. Their functions in formally detecting outliers and highly influential cases are discussed and easy computational methods are presented. An example and simulation study are presented to show the effectiveness of these statistics.

When deriving the distribution of our test statistics, we assume that the errors are Gaussian processes. The difficulty of checking multivariate normality is well known in multivariate analysis. Like many other procedures, the chi-square Q-Q plot provides only a necessary but useful check. It can capture non-normality and outliers in the $L_{2}$ sense.

Although one can compute the distribution of a linear combination of chi-squares by numerical integration as in Imhof (1961) or by simulation, we use the Satterthwaite approximation here. Our various simulations, as well as Box (1954), indicate that the Satterthwaite approximation is satisfactory for our purpose. For example, the simulated sizes were well around the significance levels when the true adjustment factor was used. Satterthwaite (1941, 1946) and Box (1954) suggested that the Satterthwaite approximation is fairly good when both of the following conditions are met: (i) all coefficients of the chi-square random variables have the same sign and (ii) all chisquare random variables have the same degrees of freedom. Note that both conditions are always 
met here; therefore, we recommend the use of Satterthwaite approximation in practice.

\section{Acknowledgments}

The authors thank the editor, an associate editor, and two referees for their comments.

\section{APPENDIX: PROOFS}

\section{Proof of (10)}

It is known in scalar regression (see Montgomery 2005, p. 397) that

$$
y_{i}(t)-\tilde{y}_{i}(t)=\left(1-h_{i i}\right)^{-1}\left(y_{i}(t)-\hat{y}_{i}(t)\right)=\left(1-h_{i i}\right)^{-1} \hat{\epsilon}_{i}(t)
$$

Combining (9) and (14) yields

$$
J_{i}^{2}=\frac{\left\|\hat{\epsilon}_{i}\right\|^{2}}{\left(1-h_{i i}\right) \hat{\Delta}_{(i)}^{2}} .
$$

Let $\hat{\sigma}(t)^{2}$ and $\hat{\sigma}_{(i)}(t)^{2}$ be the estimates of variance at time $t$ with and without the $i$ th case, respectively. It is known in scalar regression (see Montgomery 2005, p. 398) that

$$
(n-p-1) \hat{\sigma}_{(i)}(t)^{2}=(n-p) \hat{\sigma}(t)^{2}-\left(1-h_{i i}\right)^{-1} \hat{\epsilon}_{i}(t)^{2} .
$$

Then

$$
\begin{gathered}
r s s_{(i)}=(n-p-1) \int \hat{\sigma}_{(i)}(t)^{2} d t=(n-p) \int \hat{\sigma}(t)^{2} d t-\left(1-h_{i i}\right)^{-1} \int \hat{\epsilon}_{i}(t)^{2} d t=r s s-\left(1-h_{i i}\right)^{-1}\left\|\hat{\epsilon}_{i}\right\|^{2} . \\
\hat{\Delta}_{(i)}^{2}=\frac{r s s_{(i)}}{n-p-1}=\frac{(n-p) \hat{\Delta}^{2}-\left(1-h_{i i}\right)^{-1}\left\|\hat{\epsilon}_{i}\right\|^{2}}{n-p-1}=\frac{\left(n-p-S_{i}^{2}\right) \hat{\Delta}^{2}}{n-p-1}
\end{gathered}
$$

Finally, combining (15), (16) and (6) yields

$$
J_{i}^{2}=\frac{\left\|\hat{\epsilon}_{i}\right\|^{2}}{\left(1-h_{i i}\right)\left(n-p-S_{i}^{2}\right) \hat{\Delta}^{2} /(n-p-1)}=\frac{(n-p-1) S_{i}^{2}}{n-p-S_{i}^{2}} .
$$

\section{Proof of (13)}

Let $\hat{\sigma}(t)^{2}$ be the estimate of variance at time $t$ with all cases. Let $d_{i}(t)=p^{-1}\left(\hat{\mathbf{Y}}_{(i)}(t)-\right.$ $\hat{\mathbf{Y}}(t))^{T}\left(\hat{\mathbf{Y}}_{(i)}(t)-\hat{\mathbf{Y}}(t)\right) \hat{\sigma}(t)^{-2}$ be the Cook's distance at time $t$. The scalar version of (13) indicates that $d_{i}(t)=p^{-1} h_{i i}\left(1-h_{i i}\right)^{-2} \hat{\epsilon}_{i}(t)^{2} \hat{\sigma}(t)^{-2}$. Therefore, $\left(\hat{\mathbf{Y}}_{(i)}(t)-\hat{\mathbf{Y}}(t)\right)^{T}\left(\hat{\mathbf{Y}}_{(i)}(t)-\hat{\mathbf{Y}}(t)\right)=h_{i i}(1-$ $\left.h_{i i}\right)^{-2} \hat{\epsilon}_{i}(t)^{2}$. Then by (12) and (6),

$$
D_{i}=\frac{h_{i i}\left(1-h_{i i}\right)^{-2} \int \hat{\epsilon}_{i}(t)^{2} d t}{p \cdot r s s /(n-p)}=\frac{1}{p} \frac{h_{i i}}{1-h_{i i}} S_{i}^{2} .
$$




\section{REFERENCES}

Box, G. E. P. (1954), "Some Theorems on Quadratic Forms Applied in the Study of Analysis of Variance Problems, I. Effect of Inequality of Variance in the One-Way Classification," The Annals of Mathematical Statistics, 25, 290-302.

Eubank, R. L. (2000), "Testing for No Effect by Cosine Series Methods," Scandinavian Journal of Statistics, 27, 747-763.

Fan, J., and Lin, S.-K. (1998), "Tests of Significance When Data are Curves," Journal of the American Statistical Association, 93, 1007-1021.

Faraway, J. J. (1997), "Regression Analysis for a Functional Response," Technometrics, 39, 254261.

Imhof, J. P. (1961), "Computing the Distribution of Quadratic Forms in Normal Variables," Biometrika, 48, 419-426.

Montgomery, D. C. (2005), Design and Analysis of Experiments, 6th ed, New York: Wiley.

Nair, V. N., Taam, W., and Ye, K. Q. (2002), "Analysis of Functional Responses From Robust Design Studies," Journal of Quality Technology, 34, 355-370.

Ramsay, J. O., and Dalzell, C. J. (1991), "Some Tools for Functional Data Analysis," Journal of the Royal Statistical Society Series B, 53, 539-572.

Ramsay, J. O., and Silverman, B. W. (2002), Applied Functional Data Analysis: Methods and Case Studies, New York: Springer.

Ramsay, J. O., and Silverman, B. W. (2005), Functional Data Analysis, 2nd ed, New York: Springer.

Satterthwaite, F. E. (1941), "Synthesis of Variance," Psychometrika, 6, 309-316.

Satterthwaite, F. E. (1946), "An Approximate Distribution of Estimates of Variance Components," Biometrics Bulletin, 2, 110-114.

Sen, A., and Srivastava, M. (1990), Regression Analysis: Theory, Methods, and Applications, New York: Springer. 
Shen, Q., and Faraway, J. (2004), "An F Test for Linear Models With Functional Responses," Statistica Sinica, 14, 1239-1257.

Weisberg, S. (1985), Applied Linear Regression, 2nd ed, New York: Wiley. 

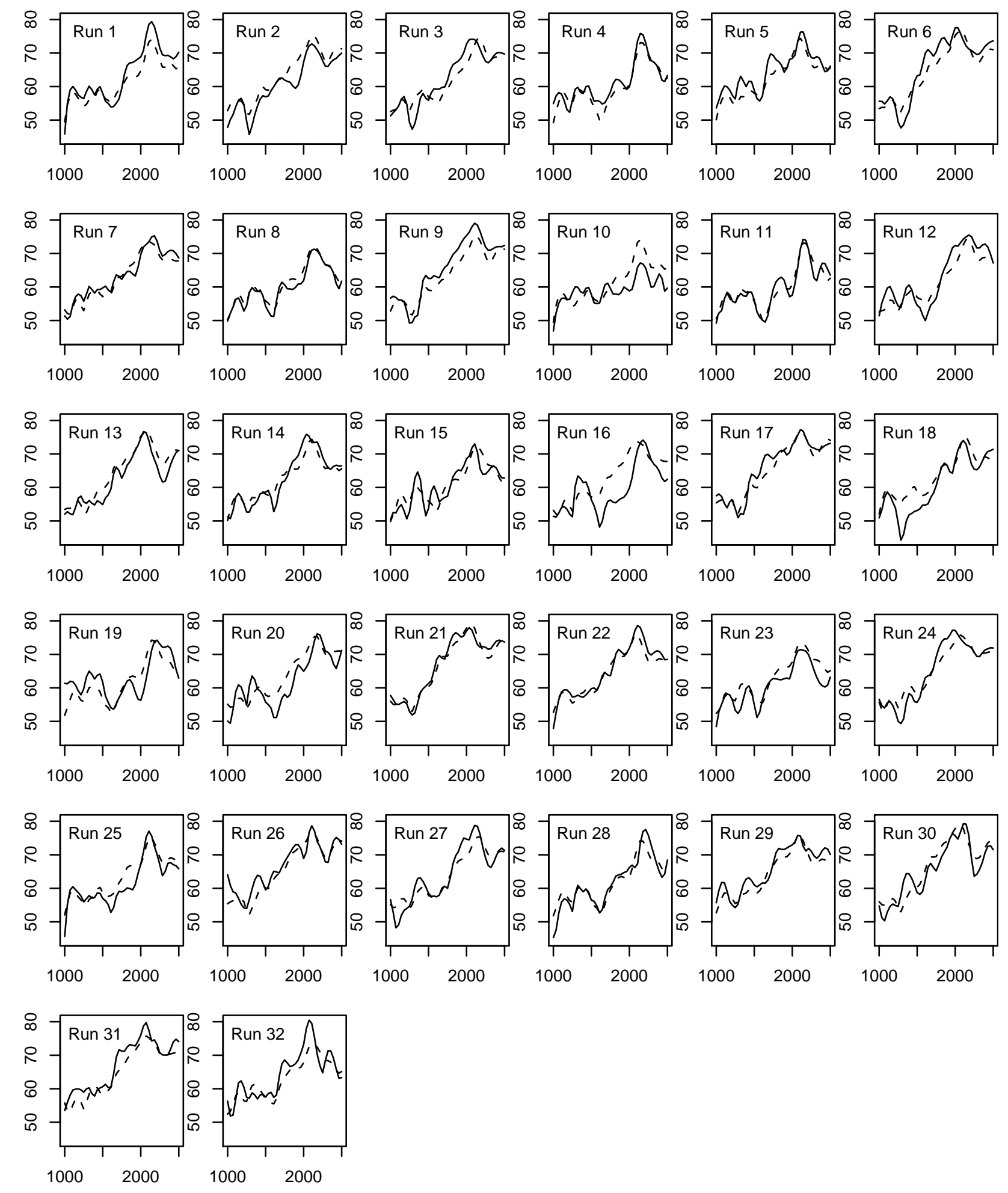

Figure 1: Observed and Fitted Response Curves. Solid lines are for observed and dashed lines are for fitted from the reduced model. 
Residuals vs Fitted

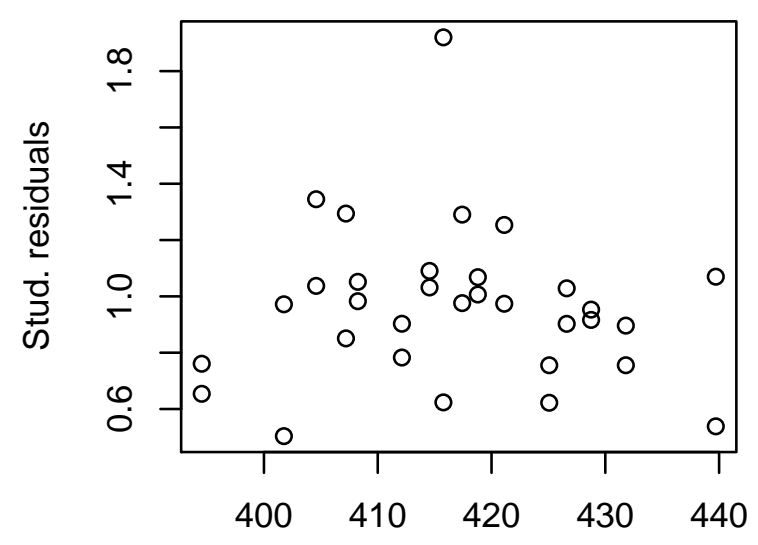

Fitted

Jackknife residuals plot

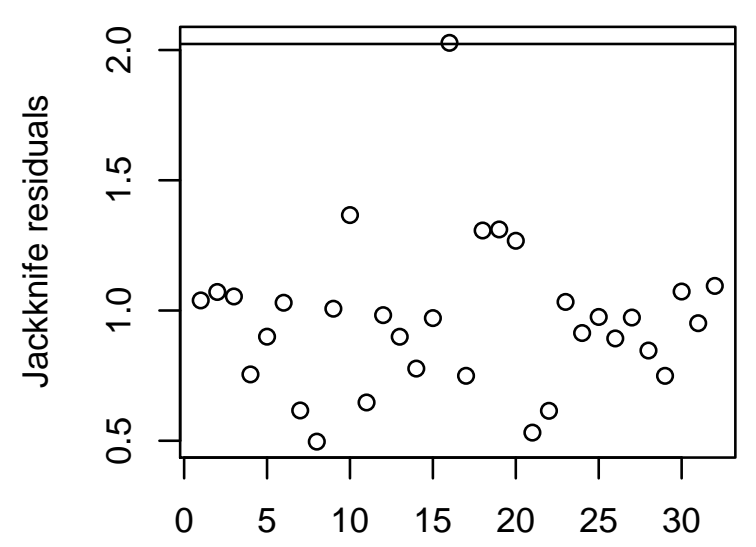

Obs. number
Chi-square Q-Q plot

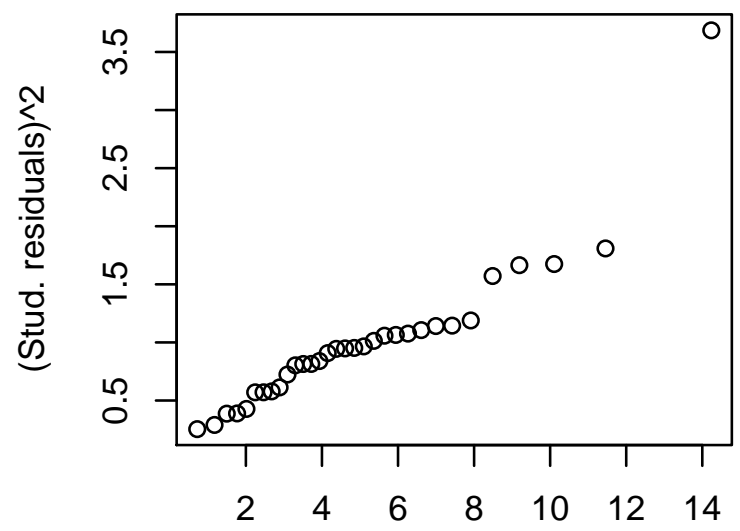

Chi-square(5.14) quantiles

Cook's distance plot

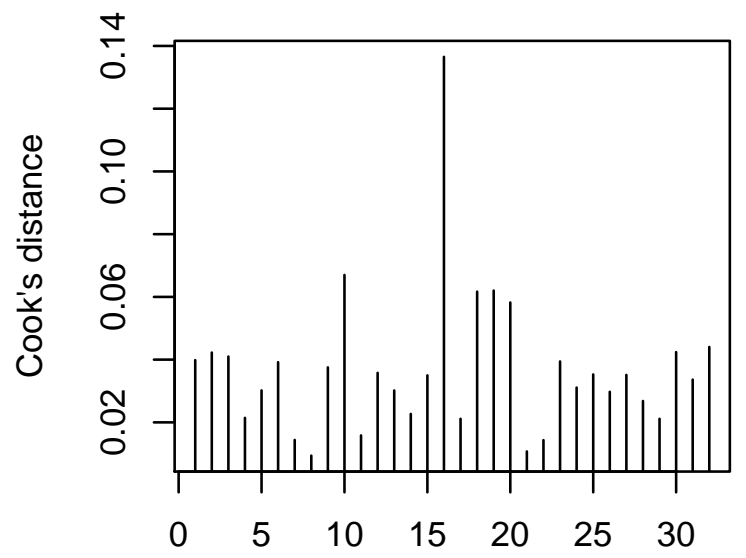

Obs. number

Figure 2: Diagnostics Plots for the Reduced Model 


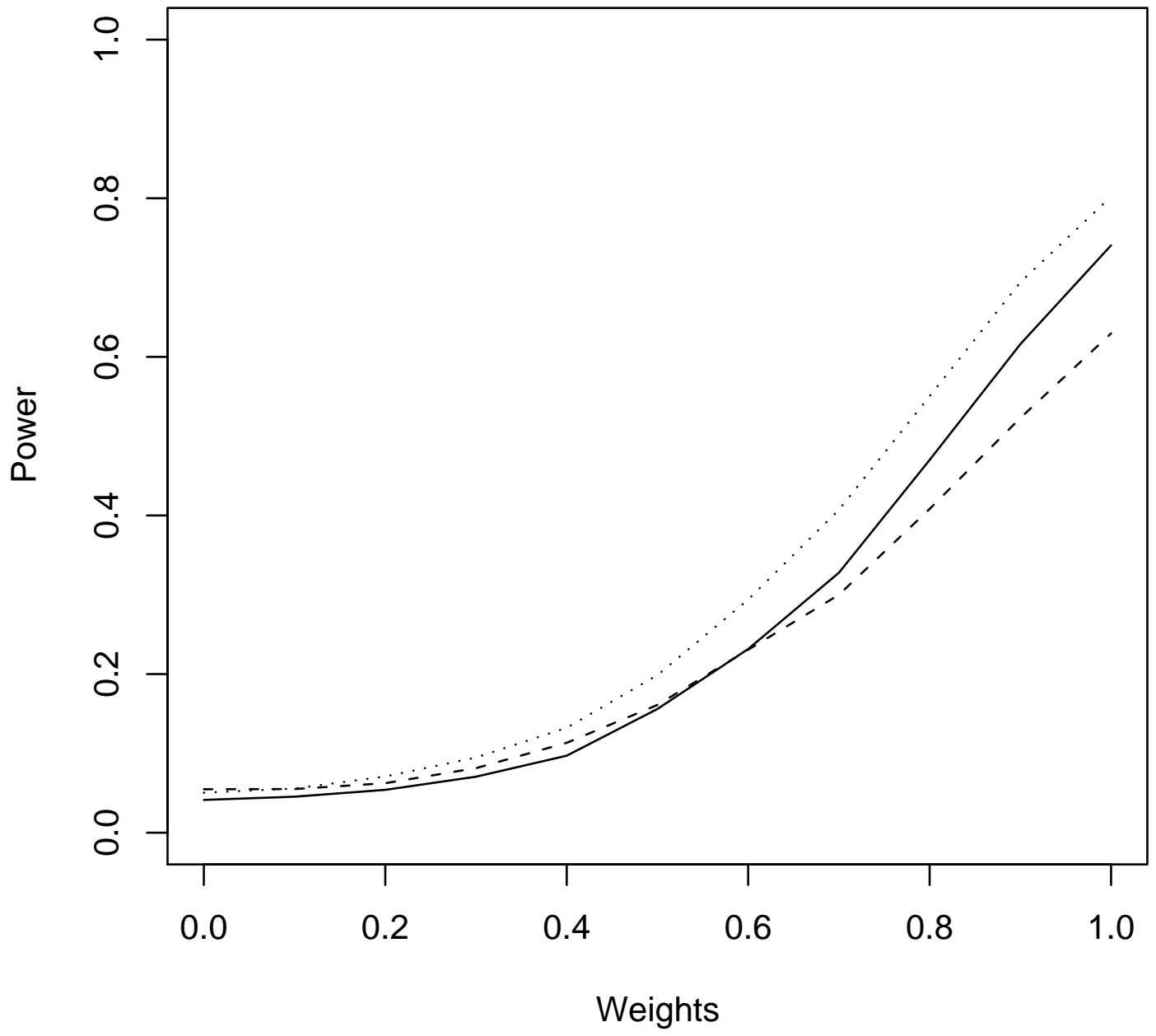

Figure 3: Simulated Power Curves of the F Test and Two B-Spline Based Tests. F test (solid), likelihood ratio tests with 5 B-spline basis functions (dashed) and with 8 B-spline basis functions (dotted). 


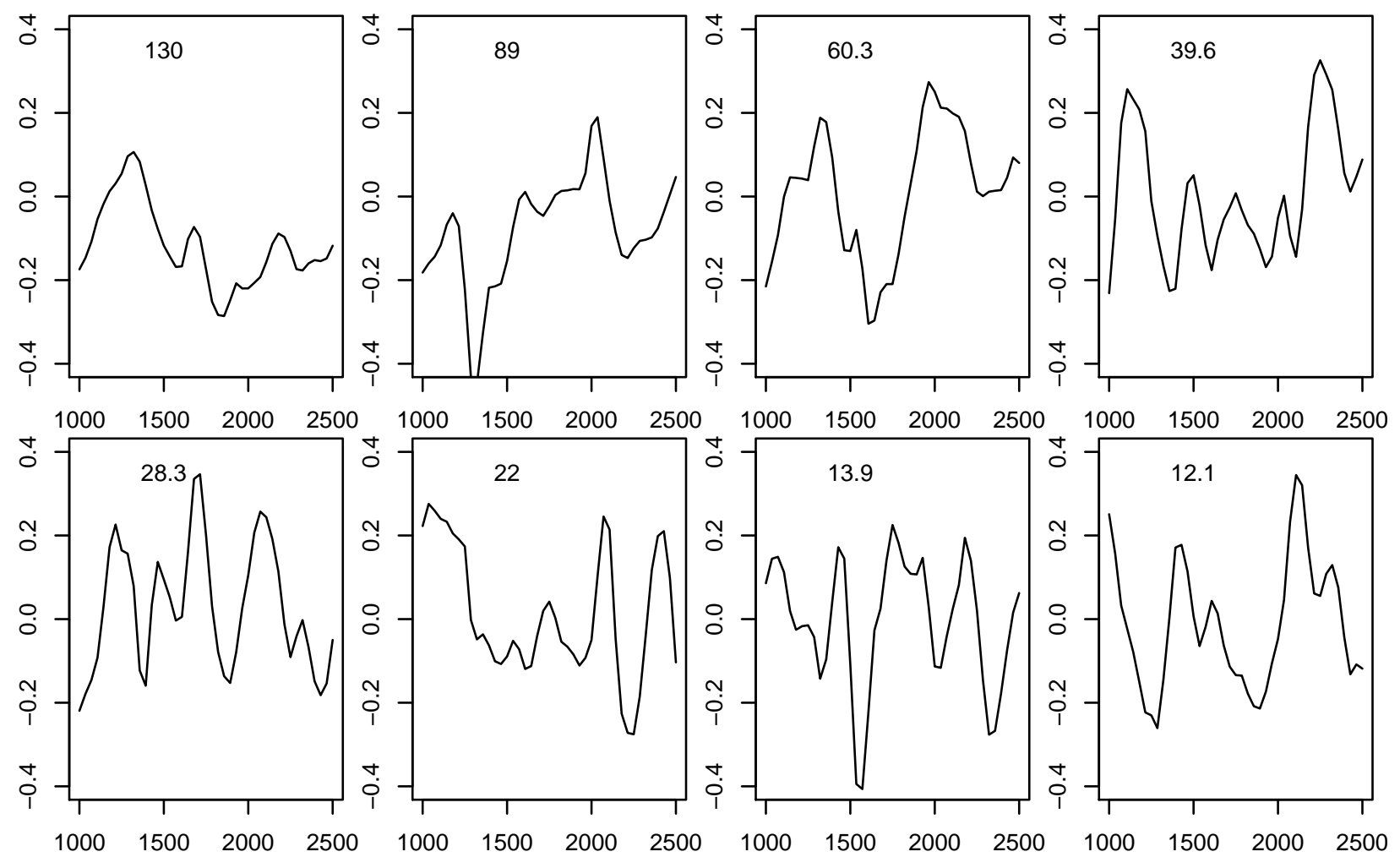

Figure 4: Estimated Eigenfunctions From the Reduced Model Without Case 16. Estimates of the first 8 eigenfunctions with the associated eigenvalues marked on the plot. 